\title{
INCLUSION BETWEEN GENERALIZED STUMMEL CLASSES AND OTHER FUNCTION SPACES
}

\author{
Nicky K. Tumalun, Denny I. Hakim and Hendra Gunawan
}

Abstract. We refine the definition of generalized Stummel classes and study inclusion properties of these classes. We also study the inclusion relation between Stummel classes and other function spaces such as generalized Morrey spaces, weak Morrey spaces, and Lorentz spaces. In addition, we show that these inclusions are proper. Our results extend some previous results in [2, 13].

Mathematics subject classification (2010): 42B35, 46E30.

Keywords and phrases: Generalized Stummel classes, generalized Morrey spaces, generalized weak Morrey spaces, Lorentz spaces.

\section{REFERENCES}

[1] M. AizenMAN AND B. Simon, Brownian motion and Harnack's inequality for Schrödinger operator, Comm. Pure. Appl. Math. 35 (1982), 209-273.

[2] R. E. Castillo, J. C. Ramos-Fernandes, and E. M. Rojas, Properties of scales of Kato classes, Bessel potentials, Morrey spaces, and weak Harnack inequality for nonnegative solution of elliptic equations, J. Diff. Equat. 92 (2017), 1-17.

[3] F. ChiarenZa, E. Fabes, AND N. GAROFAlo, Harnack's inequality for Schrödinger operators and the continuity of solutions, Proc. Amer. Math. Soc. 98 (1986), 415-425.

[4] E. B. DAVIS AND A. HinZ, Kato class potentials for higher order elliptic operators, J. London Math. Soc. 58 (1998), 669-678.

[5] G. Di FAZIo, Hölder continuity of solutions for some Schrödinger equations, Rend. Sem. Mat. Univ. Padova 79 (1988), 173-183.

[6] Eridani and H. Gunawan, Stummel classes and Morrey spaces, Southeast Asian Bull. Math. 29 (2005), 1053-1056.

[7] L. Grafakos, Classical Fourier Analysis, 2nd ed., Springer, New York, 2008.

[8] H. Gunawan, E. Nakai, Y. Sawano, And H. Tanaka, Generalized Stummel class and Morrey spaces, Publ. Inst. Math. (New Series) 92(106) (2012), 127-138.

[9] H. Gunawan, D. I. HaKim, K. M. Limanta, AND A. A. Masta, Inclusion properties of generalized Morrey spaces, Math. Nachr. 290 (2017), 332-340.

[10] A. MонAмED, Weak Harnack's inequality for nonnegative solutions of elliptic equations with potential, Proc. Amer. Math. Soc. 129 (2001), 2617-2621.

[11] C. B. Morrey, On the solutions of quasi-linear elliptic partial differential equations, Trans. Amer. Math. Soc. 43 (1938), 126-166.

[12] L. Pick, A. Kufner, O. John, And S. FučIK, Function Spaces, Vol. 1, 2nd ed., De Gruyter, 2013.

[13] M. A. Ragus A And P. Zamboni, A potential theoretic inequality, Czech. Mat. J. 51 (2001), 55-56.

[14] S. S AmKo, Morrey spaces are closely embedded between vanishing Stummel class, Math. Ineq. Appl. 17 (2014), 627-639.

[15] R. L. Wheeden And A. Zygmund, Measure and Integral: An Introduction to Real Analysis, 2nd ed., CRC Press, Boca Raton, 2015.

[16] P. ZAmBoni, Unique continuation for nonnegative solutions of quasilinear elliptic equations, Bull. Austral. Math. Soc. 64 (2001), 149-156. 